Revue d'histoire de l'Amérique française

REVUE D.HISTOIRE DE L'AMÉRIQUE FRANÇAISE

\title{
Denonville et les galériens iroquois
}

\section{W. J. Eccles}

Volume 14, numéro 3, décembre 1960

URI : https://id.erudit.org/iderudit/302064ar

DOI : https://doi.org/10.7202/302064ar

Aller au sommaire du numéro

Éditeur(s)

Institut d'histoire de l'Amérique française

ISSN

0035-2357 (imprimé)

1492-1383 (numérique)

Découvrir la revue

Citer cet article

Eccles, W. J. (1960). Denonville et les galériens iroquois. Revue d'histoire de l'Amérique française, 14(3), 408-429. https://doi.org/10.7202/302064ar d'utilisation que vous pouvez consulter en ligne.

https://apropos.erudit.org/fr/usagers/politique-dutilisation/ 


\section{DENONVILLE ET LES GALÉRIENS IROQUOIS}

Durant l'été de 1687 le Marquis de Denonville, gouverneur de la Nouvelle-France, et Jean Bochart de Champigny, l'intendant, déclanchèrent un assaut de grande envergure contre les Iroquois. Au cours de la campagne quelque deux cent vingt prisonniers furent capturés et quelques-uns furent alors envoyés en France où on les conduisit à Marseille pour service sur les galères. Les historiens ont depuis lors blâmé cette façon d'agir et les circonstances qui l'ont entourée. Leurs interprétations des événements ne tiennent pas compte cependant de tous les documents disponibles et s'appuient, dans une mesure effarante, sur une acceptation très peu judicieuse de quelques preuves plutôt douteuses. Une nouvelle interprétation de cet épisode semblerait donc nécessaire depuis longtemps.

Le récit ordinairement accepté des faits est que, en 1687, Denonville projetait de déclancher une campagne de grande envergure contre les Senecas, la plus puissante des nations iroquoises, et que, avant cette campagne, il avait reçu des ordres de Louis XIV de capturer, au cours de celle-ci, autant d'Iroquois qu'il le pourrait et de les envoyer en France afin de les faire servir sur les galères. On accuse cependant Denonville d'avoir, à dessein, capturé perfidement d'autres Iroquois moins défiants, sachant que ce serait difficile, sinon impossible, de s'emparer de quelquesuns de ces hostiles Senecas. De plus, afin d'assurer le succès de sa campagne, Denonville aurait soigneusement fait en sorte de cacher, aussi longtemps que possible, aux Iroquois, ses intentions. Ainsi lorsque le Père Lamberville, un jésuite missionnaire chez les Onondagas, vint à Québec en 1686, Denonville lui dissimula son intention de faire la guerre aux Iroquois l'année suivante; et il demanda plutôt à Lamberville de persuader autant de chefs iroquois qu'il le pourrait de se rendre l'été suivant au Fort Frontenac pour des pourparlers de paix. On soutient qu'en agissant ainsi Denonville exposait sans pitié Lamberville à une mort 
affreuse aux mains des Iroquois s'ils venaient à découvrir la mauvaise foi du jésuite.

L'année suivante, prétend-on, le Père Lamberville, pour s'acquitter de sa tâche, persuada quarante des principaux chefs de tous les villages iroquois de l'accompagner au Fort Frontenac, les assurant que Denonville lui avait promis qu'on ne leur ferait aucun mal; mais lorsqu'ils arrivèrent, les soldats français se saisirent d'eux et les mirent aux fers. Dans l'intervalle, l'armée, alors qu'elle était en route vers le fort, avait capturé quelques prisonniers et les avait envoyés à Montréal pour qu'on les y emprisonne. L'intendant, qui avait précédé le gros de l'armée, invita alors tous les Iroquois des alentours à se rendre au fort pour un festin. Lorsqu'ils eurent accédé à ce désir et qu'ils furent à l'intérieur de la palissade, on s'empara d'eux et on les attacha à des pieux. Puis, lorsque l'armée arriva au fort, Denonville envoya un de ses officiers avec un détachement de la milice canadienne s'emparer de tous les Iroquois d'un autre village situé à proximité. La majorité de ces Iroquois, affirme-t-on, appartenaient aux villages neutres de Kenté et de Ganneious et ils avaient toujours été amis des Français. Quelques mois plus tard, les hommes furent envoyés à Marseille pour servir sur les galères. Les Iroquois, prétend-on, se sentirent outragés et finalement, après avoir demandé le retour des prisonniers, auraient déclanché, en représailles, une attaque acharnée contre les établissements de Lachine. Sur ce, Louis XIV ordonna la libération et le renvoi des galériens iroquois au Canada, mais plusieurs d'entre eux avaient déjà succombé au dur traitement qu'ils avaient reçu. ${ }^{1}$

1 Père de Charlevoix, Histoire et Description Générale de la Nouvelle France, (Paris, 1744), II: 343; François-Xavier Garneau, Histoire du Canada, (6e édition, 1920), I: 328-330; J.-B.-A. Ferland, Cours d'Histoire du Canada, (Québec, 1865), II : 157-158; William Kingsford, The History of Canada, (Toronto, 1888), II : 80; Francis Parkman, Count Frontenac and New France under Louis XIV, (Toronto, 1901. D'abord édité en 1877), 136-143; Henri Lorin, Le comte de Frontenac, (Paris, 1895), 331-333; Charles W. Colby, The Fighting Governor, (Toronto, 1915), 106-107; Morden H. Long, A History of the Canadian People, (Toronto, 1942), I: 320 ; William D. Le Sueur, Count Frontenac, (Toronto, 1926), 214-215; Dr. Richard A. Preston \& Dr. Léopold Lamontagne, Royal Fort Frontenac, (Champlain Society Publications, Toronto, 1958), 47-51; George M. Wrong, The Rise and Fall of New France, (Toronto, 1928), II : 503-505. 
Voilà pour la version courante. Il reste maintenant à vérifier jusqu'où elle est, ou n'est pas, conforme aux faits.

En premier lieu, la chose n'est pas contestable, Denonville reçut de Versailles l'ordre de capturer autant de prisonniers iroquois que possible pour servir sur les galères, et il est vrai qu'il envoya en France quelques-uns des prisonniers iroquois capturés pendant la campagne, sachant qu'ils seraient envoyés aux galères. Mais il n'est pas assuré qu'aucun de ces Iroquois fût délibérément capturé à cette fin, pour la simple raison que Denonville a bien pu n'avoir reçu ces ordres qu'après son retour avec l'armée à Montréal, à la fin de la campagne. ${ }^{2}$ Ainsi il ne peut être catégoriquement affirmé que «... [Denonville] avait reçu des ordres de la cour que, étant donné que les Iroquois étaient forts et vigoureux, il devait en capturer autant que possible et les envoyer en France comme galériens. Cet ordre, sans doute, faisait allusion aux prisonniers pris en temps de guerre; mais Denonville, sachant que les hostiles Iroquois ne se capturaient

2 La dépêche ordonnant à Denonville d'envoyer en France, pour servir sur les galères, tout Iroquois qu'il capturerait au cours de la prochaine campagne, était datée du 30 mars 1687. Les vaisseaux venant de France arrivèrent à la colonie, cette année-là, au début de juin, après une traversée particulièrement rapide de trente-trois jours; ils emmenaient huit cents Troupes de la Marine. Le Chevalier de Vaudreuil, désigné pour commander les troupes régulières de la colonie, faisait partie du convoi. Lorsqu'on jeta l'ancre au Cap Tourmente il se hâta vers la ville dans la chaloupe du bateau, y arrivant au moment où Champigny était sur le point de partir pour Montréal avec la milice locale. Champigny ne différa pas son départ pour cela, et, quoiqu'il fût très fatigué et qu'il n'eût pas ses effets personnels, Vaudreuil accompagna l'intendant afin de prendre part à l'expédition. La dépêche de Champigny ne fait aucune mention du fait que Vaudreuil avait pris avec lui, en débarquant des vaisseaux, les dépêches de la cour, et la preuve négative nous porte à penser qu'il ne le fit pas. Si, comme il semble probable, les dépêches furent apportées par le convoi, il est possible qu'elles furent expédiées à Montréal afin d'atteindre Denonville et Champigny avant leur départ pour le lac Ontario avec l'expédition; mais il est tout aussi vraisemblable, dans les circonstances, que ces dépêches n'aient pas été expédiées; et de nouveau, la preuve négative indique que très probablement Denonville ne reçut pas les dépêches avant son retour de la campagne seneca. Quoi qu'il en soit, avant que l'on puisse condamner Denonville pour avoir à dessein capturé les Iroquois, conformément aux ordres de pourvoir la Marine française de galériens, il faut d'abord établir qu'il avait antérieurement reçu ces ordres; dans les circonstances, on ne peut pas présumer qu'il les avait reçus. 
pas facilement, résolut de prendre au piège leurs confiants parents. $\gg^{3}$

On ne doit évidemment pas oublier, dans aucun examen des actes et mobiles de Denonville, que ces événements eurent lieu au cours d'opérations militaires et que l'on était au dix-septième siècle, non au récent dix-neuvième siècle, ni de nos jours. Ainsi, les événements doivent être examinés à la lumière des us et coutumes de ce temps-là et non de ceux de quelque autre époque. En 1687 on se souvenait encore de la Guerre de Trente Ans; plus récemment, l'armée française, sous Louis et sous Louvois, avait acquis une réputation peu enviable de brutalité aux PaysBas et sur le Rhin; et en France même les dragonnades venaient tout juste de cesser. Si un ennemi européen civilisé et des citoyens français étaient traités de la sorte, c'était beaucoup demander que d'espérer une plus grande sollicitude envers un ennemi nullement civilisé qui ne connaissait rien des lois de la guerre, mais qui se battait seulement pour triompher par n'importe quel moyen et qui, d'habitude, soumettait ses prisonniers aux tortures les plus raffinées et les plus prolongées pour, à la fin, les manger. Denonville, par conséquent, n'envoya pas de déclaration officielle de guerre aux Iroquois; il estimait que les actes hostiles des Iroquois, au cours des années précédentes, rendaient une intervention militaire nécessaire pour les empêcher de chasser les Français de l'ouest; car tel était évidemment leur but. Contre un tel ennemi, et avec les faibles ressources à sa disposition, Denonville estimait que sa seule chance de succès était de faire avancer son armée le plus près possible des villages senecas avant qu'ils ne puissent s'assurer de ses intentions. ${ }^{4}$ Au surplus le gouvernement de Versailles lui avait conseillé de déclancher une attaque surprise puisque, dans le passé, les Iroquois avaient toujours employé ces tactiques contre les Français. ${ }^{5}$

Il est aussi tout à fait vrai que Denonville ne révéla pas au Père Lamberville son intention d'attaquer en force les Senecas

3 Francis Parkman, op. cit., 140.

4 Paris, Archives Nationales, Colonies, Séries C11A, VIII: 59-63, Denonville au Ministre, Mtl, 12 juin 1686.

5 Paris, Archives Nationales, Colonies, Séries B, XII: 30, Memoire du Roy au Sr de Denonville, Versailles, 31 mai 1686. 
et qu'il demanda au missionnaire d'inviter les chefs de toutes les nations iroquoises à le rencontrer à Cataracouï l'été suivant pour y discuter leurs affaires. Il agit ainsi, expliqua-t-il plus tard au ministre, afin de permettre au Père Lamberville de sortir en toute sécurité du village onondaga avant que le pays iroquois fût envahi, et aussi afin d'obvier au danger qu'ils découvrent ses intentions et déclanchent un assaut contre la colonie avant qu'il ne puisse les attaquer. "Il me fasche fort de le voir exposé, écrit-il au ministre, mais si Je le retirois cette année sans doute que l'orage tomberoit sur nous des cette année, car ils s'assureroient de nos desseins par sa retraite. ${ }^{6}$ Il ne croyait pas, non plus, a-t-il déclaré, que plusieurs, ni même aucun iroquois, suivraient Lamberville au Fort Frontenac; il suffirait, espérait-il, que le missionnaire se pût retirer en toute sécurité des villages lroquois. ${ }^{7}$ Le but évident de Denonville, en ne révélant pas ses projets à Lamberville, c'était donc d'assurer le salut de la colonie, ce qui était, pour lui, gouverneur, sa suprême responsabilité; et son intention, en lui demandant de persuader les chefs iroquois de l'accompagner au Fort Frontenac, était, non de les faire prisonniers, mais de faire sortir en toute sécurité Lamberville d'Onondaga. Ni l'un ni l'autre motif ne peut être considéré comme déshonorant. ${ }^{8}$ Aussi bien, en mars suivant, le ministre pouvait écrire à Denonville: «Sa Maté a approuvé la convocation que led. Sr de Denonville a faite des Nations Iroquoises à Cataracouï pour faire en sorte de retirer le Père de Lamberville. ${ }^{9}$

Le 13 juin 1687, l'armée, commandée par Denonville et par Hector de Callières et le Chevalier de Vaudreuil en sous-ordre, quitta Montréal. L'intendant les accompagnait. Lorsque le gros de l'armée eût passé les Rapides des Cèdres, Champigny prit les

6 Paris, Archives Nationales, Colonies, Séries C11A, VIII : 120, Memoire de l'Estat Present des Affaires de Canada et des Necessitez de faire la guerre l'an prochain aux Iroquois. A Quebec le $8^{\text {me }} 9^{\text {re }} 1686$. Joint a la lettre de M. de Denonville le $89^{\text {re }} 1686$.

7 Ibid.

8 Mais voir Preston \& Lamontagne, op. cit., 48: «sachant ce qui arriverait le Gouverneur s'inquiétait à la pensée que le Père Lamberville s'échapperait difficilement des mains des Iroquois, mais il ne fit rien pour le protéger. 》

9 Paris, Archives du Ministère des Affaires étrangères, Mémoires et Documents, V-3: 283, Réponses aux Lettres de Canada, 30 mars 1687. 
devants avec trente hommes dans quinze canots chargés de vivres, afin de s'assurer que tout serait prêt à Cataracouï pour recevoir l'armée et faire en sorte qu'elle pût se remettre en route vers le pays ennemi dans le plus court délai. ${ }^{10}$ Pendant les quelques jours qui suivirent, quatre groupes d'Iroquois, distincts et séparés les uns des autres, furent faits prisonniers; il importe de tenir compte des différentes circonstances qui entourent la capture de chacun de ces groupes. C'est pour n'avoir pas tenu compte de ces circonstances qu'on commît dans le passé tant de confusion.

Pendant que l'armée franchissait péniblement la longue suite de rapides, Denonville se précautionna contre un guet-apens possible; car une telle attaque, déclanchée avec violence, aurait pu occasionner de très lourdes pertes et peut-être obliger l'expédition à retourner en arrière. ${ }^{11}$ Ainsi, il envoya un groupe d'éclaireurs devant le gros de l'armée et celui-ci captura trois Iroquois à Châteauguay. ${ }^{12}$ Le 19 juin, un canot, revenant du fort, rapporta qu'un chef et quelques autres cayugas avaient été vus dans les environs. Un détachement fut envoyé pour les capturer et il revint avec quatre hommes, leurs femmes et deux jeunes garçons. Deux de ces hommes étaient des chefs cayugas importants et très hostiles aux Français. L'un d'eux, Oréaoué, avait contribué plus tôt au pillage de canots français et il avait gravement maltraité le Père Carheil. ${ }^{13}$ Le lendemain tous ces prisonniers furent

10 Paris, Archives Nationales, Colonies, Séries C11A, IX: 108, Memoire du Voyage Pour l'Entreprise de M. le Marquis de Denonville Contre les Sonontouans Ennemis de la Colonie, Selon les ordres du Roy. Par le mesme M. de Denonville [Qué., 1687]; 33-36, Champigny au Ministre, Qué., 16 juillet 1687; 26, Denonville au Ministre, Ville Marie, 8 juin 1687. (L'affirmation de Preston \& Lamontagne, op. cit., 47, à savoir que la manœuvre de l'intendant «...e était seulement un prétexte pour cacher l'action la plus lâche perpétrée en Amérique du Nord par les Français 》, manque totalement de preuve.)

${ }_{11}$ Archives publiques du Canada, Documents St-Sulpice, Paris, Registre 25, Campagne de M. le Marquis de Denonville dans le Pays des Iroquois; le chevalier de Baugy, Journal d'une Expédition contre les Iroquois en 1687, (Paris, 1883), 90-91.

12 Ibid., 61.

13 Ibid., 65, 68; Jesuit Relations and Allied Documents, éd., R. G. Thwaites, (Cleveland, 1896-1901), LXII : 102-104, Lettre de Jean de Lamberville à Rev. Père Beschefer, Onnontagué, 25 aoust 1682 ; Memoire du Voyage Pour l'Entreprise de M. le Marquis de Denonville Contre les Sonontouans..., op. cit., 108. 
envoyés à Montréal et incarcérés. ${ }^{14}$ Le vingt-trois, un autre chef cayuga fut capturé avec trois femmes et deux enfants. ${ }^{15}$ Denonville écrivit plus tard que ce cayuga avait été envoyé pour donner avis de la marche de l'armée française, et, lorsqu'on l'interrogea, le prisonnier affirma qu'Oréaoué et sa troupe étaient en route vers Montréal pour voir ce qui s'y passait; il affirma aussi qu'ils avaient espéré capturer quelques Français en s'en retournant. ${ }^{16}$ Et l'aide de camp de Denonville, le chevalier de Baugy, déclare: «[Lamberville] nous assura que les quatre premiers Onontagués et le chef des Goyogouins qui avoient esté pris, n'estoient venus que pour nous épier; ce qui est positif, est que l'on trouva dans le sac du chef Goyogouin un lien qu'ils ne portent avec eux que pour faire des Prisonniers. »17 Les Français considérèrent donc les dix-neuf iroquois, capturés par l'armée alors qu'elle était en route vers Cataracouï, comme des espions envoyés pour surveiller les mouvements de l'armée. Il serait, par conséquent, juste de considérer ce premier groupe comme de légitimes prisonniers de guerre et il n'est pas juste de qualifier leur capture de perfidie. $^{18}$

Le 24 juin, un canot, envoyé de Cataracouï par Champigny, rapporta que plusieurs Iroquois pêchaient à l'île d'Otoniata et le

14 Ibid.

15 Ibid., 109-110.

16 Ibid.

17 Le chevalier de Baugy, op. cit., 77-78.

18 Francis Parkman (op. cit., 141) affirme de ces captifs iroquois en particulier «... qu'ils avaient tous leurs femmes et leurs enfants avec eux, ce qui n'était jamais le cas des Iroquois sur le sentier de guerre. De là l'affirmation de Denonville qu'ils étaient venus avec des intentions hostiles, est très invraisemblable. 》 Parkman continue alors en disant que «il n'est pas déraisonnable de supposer $\gg$ qu'ils étaient en route vers Montréal pour $\mathrm{y}$ 《fumer le calumet de paix » à la requête de Denonville et par l'entremise de Lamberville. Parkman, cependant, se trompe tout à fait lorsqu'il présume que les Iroquois n'amenaient jamais leurs femmes et leurs enfants dans leurs expéditions de guerre. Pour ne citer que deux exemples: en 1690, lorsqu'un groupe de guerriers iroquois attaqua un détachement français qui remontait l'Outaouais, quatre prisonniers iroquois furent pris, deux hommes et deux femmes; en avril de l'année suivante, un groupe de guerriers, comprenant cent quarante hommes, quarante femmes et vingt enfants, attaqua les établissements de La Chesnaye et cinq hommes et treize femmes furent capturés par les Français. Il n'est pas non plus raisonnable de penser que ces Iroquois capturés par Denonville se rendaient à Montréal pour y parler de paix à la requête de Denonville et de Lamberville. Ils avaient été invités à aller à Cataracoui à cette fin, non à Montréal. 
long de la côte sud de l'autre côté de l'île, à quelque trente milles en aval de Cataracouï. Denonville envoya immédiatement un détachement de cent indiens alliés, commandé par le Sieur Le Moyne de Ste-Hélène, capturer ce second groupe. Le soir suivant, l'intendant envoya un message annonçant qu'il avait fait prisonniers tous les Iroquois des environs du fort afin de les empêcher d'informer l'ennemi de la marche de l'armée; il avertit aussi Denonville qu'il avait invité les Iroquois vus à Otoniata de venir au fort où on les fit tous aussi prisonniers. ${ }^{19}$ De Baugy raconte que ce dernier groupe n'était pas arrivé au fort lorsque Champigny envoya ce message, mais l'on espérait qu'il le serait lorsque Denonville apprendrait la chose. ${ }^{20}$ Denonville fit alors dire à Ste-Hélène de continuer de s'emparer, à Otoniata, de tout iroquois qui n'était pas allé au fort, et il le chargea de s'assurer qu'aucun mal ne serait fait au fils d'un chef onondaga appelé Hotreouati. ${ }^{21}$ Il renvoya aussi au fort le canot qui avait apporté cette nouvelle avec instructions d'avertir les prisonniers iroquois de Kenté qui composaient le troisième groupe - qu'on ne leur ferait aucun mal et qu'ils avaient été capturés seulement pour les empêcher d'avertir les Cinq Nations de l'arrivée de l'armée au fort. ${ }^{22}$ Deux jours plus tard, Champigny, s'en retournant à Montréal, rejoignit l'armée. Il avertit Denonville que tous les Iroquois des environs $\mathrm{du}$ fort avaient été capturés, soit cent vingt dont trente étaint des hommes et le reste des femmes et des enfants. ${ }^{23}$ Champigny et de Baugy le déclarent tous les deux: la garnison du fort ne se sentant pas en nombre suffisant pour les prendre de force, on avait invité ces Iroquois à une fête et une fois rendus à l'intérieur de la palissade, on les avait constitués prisonniers. ${ }^{24}$

Il est évident que Champigny employa un subterfuge pour capturer ces deux groupes d'Iroquois. Il est aussi évident qu'il

${ }^{19}$ Le chevalier de Baugy, op. cit., 71, 73-74; Memoire du Voyage Pour l'Entreprise de M. le Marquis de Denonville Contre les Sonontouans..., op. cit., 110.

20 Le chevalier de Baugy, op. cit., 73-74.

21 Ibid.

22 Ibid.

23 Memoire du Voyage Pour l'Entreprise de M. le Marquis de Denonville Contre les Sonontouans ..., op. cit., 111-112.

24 Ibid., 33-36, Champigny au Ministre, Qué., 16 juillet 1687; Le chevalier de Baugy, op. cit., 76-77. 
agit ainsi de sa propre initiative et Denonville n'était en aucune manière responsable de ce moyen employé par l'intendant. Quoique les Iroquois eux-mêmes employassent de tels procédés, ${ }^{25}$ on doit néanmoins considérer au pis cette façon d'agir, comme une perfidie, et, au mieux, comme un procédé très douteux. ${ }^{26}$ Cependant on ne doit pas aussi oublier que, si Champigny ne les avait pas faits prisonniers de cette façon, ils auraient été capturés par le détachement de Ste-Hélène quelques heures plus tard; ainsi leur sort aurait été le même.

Dans la soirée du 29, alors que l'armée était à moins d'une journée de marche du Fort Frontenac, le Père Lamberville la rejoignit, apparemment seul, et au grand soulagement de Denonville. $^{27}$ Les Iroquois, selon son récit, prévenus par les autorités d'Albany, connaissaient déjà l'approche de l'armée française et ses intentions. ${ }^{28}$ Le lendemain Denonville prit les devants et son premier geste, en arrivant au fort, fut de libérer et de renvoyer à Onondaga le fils et le frère de Hotreouati, qui étaient du groupe d'Otoniata. Dans sa dépêche au ministre, il déclara plus tard

25 Pour des exemples de perfidie iroquoise voir Jesuit Relations..., LIV : 74, Relation de 1669-1670; Paris, Archives du Ministère des Affaires étrangères, Séries Amérique, V: 211-212, Talon au Ministre, Qué., $1^{\text {er }}$ sept. 1666.

26 Frontenac condamna énergiquement cette action, la considérant comme une trâitrise, et il embrouilla la question par sa prétention que tous les prisonniers avaient été capturés de cette façon; il citait en particulier le chef de la tribu cayuga, Oréaoué, qui, de fait, était un des captifs cueillis par l'armée alors qu'elle se dirigeait vers Cataracoui. (Voir Paris, Archives Nationales, Colonies, Séries C11A, XI: 7, Relation de ce qui s'est passé de plus remarquable en Canada depuis ... Novembre 1689 ... jusqu'au mois de Novembre 1690. Monseignat; 132, Parole qui doit estre ditte a Loutaouac pour le dissouader de l'alliance qu'il veut faire avec Liroquois et Langlois [Frontenac], 1690.) Cependant quelques années plus tard, il tenta d'employer à peu près les mêmes méthodes dans un but semblable. En 1698, il écrivait: "Cette contrarieté de paix avec nous et de Guerre contre nos alliez, obligea M. le Comte de frontenac qui pouvoit justement douter de la sincerité des Iroquois, d'ordonner au $\mathrm{S}^{\mathbf{r}}$ de la Gemeraye de se tenir sur ses gardes, et de prendre des mésures pour s'assurer de Quelques considerables sans bruit, qui s'hazarderoient d'Entrer dans son fort, et qui pourroient servir d'otage pour les autres. 》 (Voir Paris, Archives Nationales, Colonies, Séries F3, Moreau de St-Méry, VIII: 49, Relation de ce qui s'est passé de plus remarquable en Canada... 1697... Oct. 20e 1698 [Frontenac].)

27 Memoire du Voyage Pour l'Entreprise de M. le Marquis de Denonville Contre les Sonontouans..., op. cit., 111.

28 Le chevalier de Baugy, op. cit., 77-78. 
qu'il avait relâché ces deux hommes parce que Hotreouati était bien disposé à l'égard des Français et que, au cours de l'année précédente, ce chef avait cherché à réprimer l'agressivité des Senecas et autres Iroquois. ${ }^{29}$ Le premier juillet, Denonville reçut un mot du Sieur Peré, envoyé au village de Ganneious, à quelque vingt-cinq milles du fort, pour y capturer les Iroquois. Peré disait n'avoir pas assez d'hommes pour arrêter tous ceux qui étaient là. Denonville envoya alors quarante Canadiens, aux ordres des Srs de Repentigny et Portneuf, pour aider Peré. Le trois, ils revinrent avec le quatrième groupe de prisonniers, dixhuit guerriers et quelque soixante-deux femmes et enfants. Les hommes furent tous liés ainsi que les autres hommes emprisonnés dans le fort. Le total se montait alors à cinquante-et-un hommes et à cent cinquante femmes et enfants. ${ }^{30}$

On ne saurait dire si ce quatrième groupe, les Iroquois de Ganneious, fut pris traîtreusement; ils furent capturés par les Français les armes à la main; et ceux-ci, avec le second et le troisième groupes, ceux d'Otoniata et de Kenté, furent capturés pour des raisons de sécurité militaire. Les Iroquois de Kenté et de Ganneious, fréquemment désignés sous le nom d'« Iroquois neutres », étaient pour la plupart des Cayugas qui, plusieurs années plus tôt, s'étaient fixés sur la côte nord du lac pour se protéger contre les attaques des Andastes qui, à l'époque, serraient de près les Cinq Nations. ${ }^{31}$ Denonville affirma que les Cinq Nations avaient contraint les hommes les plus alertes de ces villages de se joindre à elles, augmentant ainsi leur effectif et dépeuplant la côte nord. ${ }^{32}$ Un mémoire contemporain anonyme, écrit apparemment par un Sulpicien, dit ceci, à ce sujet: «... au passage des Rapides qui sont au dessous du lac St. François au lieu d'estre

29 Memoire du Voyage Pour l'Entreprise de M. le Marquis de Denonville Contre les Sonontouans..., op. cit., 111-112. Voir aussi à ce propos, ibid., 98-99, 26 aoust 1686, Canada. Memoire instructif des mesures que M. de Denonville a prises pour la guerre resolue contre les Iroquois au Printemps ...; 18, Denonville au Ministre, Qué., 8 mai 1686.

30 Memoire du Voyage Pour l'Entreprise de M. le Marquis de Denonville Contre les Sonontouans ...., op. cit., 112, 113.

31 Jesuit Relations and Allied Documents, LI: 176, 254-256, Relation de ce qui s'est passé dans la Nouvelle France ès années 1667 et 1668 .

32 Paris, Archives Nationales, Colonies, Séries C11A, IX: 70-71, Denonville au Ministre, Mtl, 25 aoust 1687. 
arresté comme il le craignoit par quelques Embuscades, il prit en chemin sans coups ferir plusieurs Espions Iroquois, et il s'assura aussy sans peine a Katarakouy de 120 femmes et enfants, et de 40 hommes de la meme nation qui auroient peut fortifié les ennemis, s'ils eussent receu ordres d'eux de les aller joindre et qui pouvoient dans la suitte nous servir d'ôtages pour la seureté des prisonniers qu'on feroit sur nous. »33 Si l'on tient compte de la façon dont les Iroquois traitaient leurs prisonniers et aussi que Denonville pouvait prévoir la capture de quelquesuns de ses hommes avant la fin de la campagne, ces deux considérations pouvaient justifier amplement la capture d'un aussi grand nombre que possible d'Iroquois. Denonville lui-même fait clairement entendre que tel était bien son sentiment. ${ }^{34}$

La plus grave et certainement la plus préjudiciable accusation portée contre Denonville fut celle que proféra le Père Lamberville, dans une de ses lettres à un ami missionnaire en Chine et datée de Paris, 23 janvier 1695; le Père écrit donc plus de sept ans après les événements. Sa version va tout à fait à l'encontre de faits jusqu'ici connus, et elle renferme quelques implications assez embarrassantes. Charlevoix l'utilisa d'abord sans discernement; avec son habituelle négligence, il l'employa pour donner de l'épisode un récit tronqué. Par la suite, Garneau et Ferland acceptèrent et citèrent entièrement mais inexactement la version de Charlevoix et les historiens qui suivirent ont donné des versions abrégées de Garneau et de Ferland qui ne font que répéter leurs erreurs. Voici la partie suspecte de la lettre de Lamberville:

Le gouverneur... me députa vers les Iroquois pour les inviter tous, en la personne de leurs chefs à se trouver le printemps au rendés vous qu'il marqua pour y parler de la continuation de la paix et des moyens de la bien maintenir avec eux, et eux avec luy. On me dit d'engager la foy et la parole qu'on leur donnoit de leur seureté et liberté de venir à ce rendés vous et de là de retourner chès eux. J'exécute mes ordres. J'assemblay 40 des principaux chefs de toutes les bourgades Iroquoises. Je leur donne la

33 Archives publiques du Canada, Documents St-Sulpice, Paris, Registre 25, Campagne de M. le Marquis de Denonville dans le Pays des Iroquois. 34 Denonville au Ministre, Mtl, 25 aoust 1687, loc. cit. 
parole du gouverneur. Je leur proteste qu'estant chrétien et choisy par le Roy pour estre son lieutenant général en ce païs-là, il falloit qu'ils crûssent qu'il estoit un homme incapable de manquer à sa parole ni de tromper contre le droit des gens. Sur quoy ils acquiescèrent à mes instances. Ils furent au rendès vous, ou l'on les trompa, on les mit au fers et dans les prisons. On leur pilla quantité de pelletries qu'ils avoient aportées pour marquer aux français par leur commerce qu'ils se fioient à eux. On les transporta en france. On les mit à Aix où ils sont morts de misère à la réserve de 13 , qu'on leur ramena à cause que leurs compatriotes alloient venger cette perfidie....

En premier lieu, l'affirmation de Lamberville à savoir que quarante des principaux chefs de tous les villages iroquois qui se seraient portés au Fort Frontenac à l'invitation de Denonville, auraient été faits prisonniers et plus tard envoyés en France, est erronée. De fait, seulement trente-six des prisonniers furent envoyés en France; ${ }^{36}$ la plupart d'entre eux venaient des villages de Kenté et de Ganneious ; ${ }^{37}$ quelques-uns étaient des captifs pris par l'armée avant son arrivée à Cataracoui; et Lamberville, d'après de Baugy, reconnaît ces derniers pour espions. ${ }^{38}$ Ainsi aucun de ces Iroquois paraît n'avoir été du groupe de ceux-là qui s'étaient rendus à Cataracoui sur l'ordre de Denonville; l'arithmétique seule suffit à le démontrer: Lamberville fait ici erreur. Puisque les Iroquois capturés avant l'arrivée de l'armée au fort et que les Iroquois neutres de Kenté et de Ganneious n'ont pu être pris parce qu'invités par Lamberville à se rendre au même fort, ${ }^{39}$ il s'ensuit que si on fit des prisonniers, ce ne pouvait être que les Iroquois d'Otoniata. Du reste, deux mois après cet incident, les Onondagas, les Senecas et les Cayugas

35 Jesuit Relations and Allied Documents, LXIV: 238-258, Lettre du P. Jean de Lamberville à un Père Missionnaire de Chine, Paris, 23 Jan. 1695. 36 Ibid., LXIII : 281, R.P. Beschefer à M. Cabart de Villermont, Qué., 19 Sept. 1687.

37 Denonville au Ministre, Mtl, 25 aoust 1687, loc. cit.

38 Le chevalier de Baugy, op. cit., 77-78.

39 Mais voir Preston \& Lamontagne, op. cit., 48, où il est affirmé que les prisonniers capturés par Champigny et Peré furent « la plupart d'entre eux innocemment rassemblés par le Père Lamberville et amenés de l'autre côté du Lac Ontario. \$ 
informaient très catégoriquement les juges de paix d'Albany qu'aucun des leurs n'était allé s'entretenir avec Denonville à Cataracoui ; ${ }^{40}$ et en septembre suivant les Onondagas affirmaient de nouveau: «Le Gov ${ }^{\mathrm{r}}$ du Canida nous pria de venir à Cadarachqui ce printemps pour nous y entretenir avec lui, mais Son Excell, à qui nous obéîmes, nous ordonna de ne pas y aller.» 41 Aucun document ne mentionne que des Mohawks furent impliqués de quelque manière dans l'affaire; il semblerait donc que, si quelques-uns des prisonniers iroquois envoyés en France avaient été capturés pour s'être rendus à Cataracoui, à la demande de Lamberville, en vue de s'entretenir avec Denonville, ils devaient tous être des Oneidas. Que quelques Oneidas aient été capturés, la chose n'est pas impossible. Mais si tel fut le cas, il est très étonnant que les chefs Oneidas ne s'en plaignirent pas au gouverneur de New-York. De fait, voici peut-être l'explication la plus plausible de la présence des Iroquois à Otoniata; c'était simplement un groupe de chasseurs iroquois. Cette région était, en effet, le principal territoire de chasse des Onondagas, des Oneidas et des Cayugas. Au besoin la plainte adressée, en février suivant, par les Cinq Nations de la Confédération iroquoise - laquelle comprenait les Oneidas - au gouverneur Dongan de New-York, confirmerait notre explication: «... nous désirons que les castors et autres marchandises qui furent enlevés à nos gens à Catarachqua alors que nous revenions de chasser le castor soient restitués, lesquels gens sont maintenant en France et au Canada... ${ }^{42}$ On ne peut donc affirmer d'une manière décisive qu'aucun de ces Oneidas fut capturé parce que Lamberville l'avait persuadé d'aller s'entretenir avec Denonville,

40 The Livingston Indian Records 1666-1723, ed. Lawrence $\mathrm{H}$. Leder (Gettysburg, 1956), 121-122, Propositions made by the Cayouges Sachims to ye Magistrates of Albany ye 27th day of June 1687.

41 Documents Relating to the Colonial History of New York, ed. E. B. O'Callaghan \& J. R. Brodhead (Albany, 1856-1883), III: 485, Propositions of the Onondagas to the Mayor and Common Council of Albany, Sept. 14, 1687.

42 Ibid., 536, Additional Propositions of the Six Nations to Governor Dongan, Feb. 16, 1688. 
mais il semble très invraisemblable qu'un seul le fut.. ${ }^{43}$ Ainsi les documents indiquent assez clairement que l'exposé de Lamberville n'est pas conforme aux faits.

Dans cette même lettre le Père Lamberville affirme encore deux choses : après la capture des délégués iroquois, Denonville aurait cherché à conclure un accord de paix avec les Iroquois; et alors qu'il se rendait lui-même au fort avec un autre groupe de délégués, les Français seraient allés par un autre chemin, attaquer et piller leurs villages. C'est le 29 juin cependant que Lamberville rejoignit l'armée française, à ce moment en route vers Cataracoui; les Français n'atteignirent le fort que le 1er juillet et l'attaque contre les villages senecas n'eut lieu que les 13-14 juillet; l'exposé de Lamberville est donc tout à fait à l'encontre de la réalité. En outre aucun autre document ne mentionne que Denonville chercha, à cette époque, à entamer des pourparlers de paix. Et il est inconcevable s'il avait agi ainsi, qu'on ne l'eût pas mentionné. Lamberville déclare plus loin qu'avant de quitter Onondaga pour Cataracoui avec huit chefs Iroquois, il avait reçu des lettres - il ne dit pas de qui - lui mandant de quitter le pays iroquois par quelque moyen que ce fût, parce qu'on allait déclancher une attaque contre eux. Le Père pouvait difficilement ne pas conclure, de ces lettres, que la conférence de paix proposée n'était qu'une ruse; néanmoins, selon ce qu'il en dit, il ne prévint pas ses compagnons iroquois de ce fait: il déclare, en effet, qu'ils furent informés du guet-

43 Le Chevalier de Baugy, en relatant la capture des Iroquois qui étaient à Otoniata, déclare (page 80): «Mr le Marquis a fait relascher le fils de la Grande Gueule [Hotreouati] pour luy marquer qu'il ne manque pas à sa parole, estant venus pour ce qu'il leur avoit mandé. 》 Il est difficile de savoir au juste quel sens donner à cette déclaration énigmatique. Denonville donna de toutes autres raisons pour avoir libéré ce prisonnier et son oncle: mais si la déclaration de Baugy est exacte, on pourrait alors en déduire que, puisque Denonville relâcha ce prisonnier Onondaga pour montrer qu'il était un homme de parole, il aurait alors dû relâcher pour le même motif, tout autre prisonnier dans la même situation. Puisqu'il ne relâcha que ces deux prisonniers, on pourrait alors supposer que seulement deux Iroquois se rendirent à Cataracoui à la requête de Denonville et de Lamberville, et qu'aucun des deux ne fut détenu. Mais, à l'encontre de ceci, les Onondagas affirmèrent à deux reprises qu'aucun des leurs n'était allé s'entretenir avec Denonville. Ou de Baugy ou les Onondagas se trompaient; il semblerait plus vraisemblable que ce fût de Baugy. 
apens par quelques-uns des leurs échappés du fort. Si telle était la vérité, il s'ensuivrait donc, et du propre témoignage de Lamberville, qu'entre le temps où il reçut les lettres lui ordonnant de quitter le pays iroquois et celui où ses compagnons iroquois apprirent les événements du Fort Frontenac, il se fit le complice de leur trahison; car il pouvait les avertir, mais il ne le fit pas, que l'entretien proposé n'était en réalité qu'un piège.

De toute évidence, la lettre du Père Lamberville contient un récit des événements qui ressemble peu à ce qui eut lieu réellement. Lorsqu'il écrivit sa lettre, il n'espérait pas vivre beaucoup plus longtemps; il était brisé par ses privations presque incroyables endurées dans les missions. ${ }^{44}$ De plus il éprouvait une extrême amertume au sujet de la guerre contre les Iroquois; il avait depuis des années consacré tous ses efforts à empêcher qu'elle n'éclate, sachant trop bien tout ce que signifierait une reprise des hostilités entre Français et Iroquois, tant pour les établissements français que pour ses longues et difficiles années d'effort missionnaire parmi les Onondagas. Ainsi peut-on charitablement présumer qu'à l'heure où il écrit sa lettre, ses souvenirs s'étaient fort embrouillés. Il éprouvait aussi beaucoup d'amertume, et cela se comprend, de l'envoi de quelques prisonniers iroquois aux galères. Admettons même qu'il eût vraiment invité les chefs iroquois à se rendre à Cataracoui - hypothèse qui n'a de base que son assertion écrite plus de sept ans après l'événement - ${ }^{45}$ il est fort plausible qu'avec les années, le Père en soit venu à penser que les Iroquois faits prisonniers s'étaient rendus au fort à sa demande, bien que, de fait, il semblerait qu'aucun n'agît ainsi.

44 Lettre du P. Jean de Lamberville..., op. cit., 358.

45 On trouve dans les Archives une curieuse déclaration faite à Albany en juillet 1686 par trois chefs Mohawks: «Toutes les nations s'étaient réunies à Onondage et avaient tenu conseil pendant sept jours. Et le prêtre nous demanda pourquoi nous tenions conseil, ce que d'abord nous ne voulûmes pas dire. Mais il promit de nous révéler aussi quelque chose. Nous lui dîmes alors que le Gouverneur nous avait ordonné de nous tenir tranquilles et de ne pas reprendre la hache de guerre. Il nous dit donc que lorsque le maïs serait presque mûr les Français viendraient nous tuer. Les Français se proposent aussi de vous envoyer chercher et si vous y allez, il en sera fait de vous. $\gg$ Voir The Livingston Indian Records 1666-1723, 103, Proposal of the 3 Maquase Sachems [Juillet 1686]. 
De retour à Montréal après la campagne seneca, Denonville répondit au mémoire du roi du 30 mars 1687. Dans ce mémoire, qui lui était adressé ainsi qu'à Champigny, Sa Majesté exigeait que tous les prisonniers capturés au cours de la campagne fussent envoyés en France pour servir sur les galères. La dépêche du gouverneur au ministre le fait clairement voir: ces ordres n'étaient pas de son goût; le ton même de la dépêche laisse entendre que l'idée ne lui était jamais venue qu'on pût exiger de lui une telle ligne de conduite. Ainsi il déclare:

Vous m'avez ordonné de vous envoyer les prisonniers que nous feriont, vous avez veu Monseigneur qu'il ne nous a pas esté possible d'en faire aucun aux Sonontouans et quand nous en auriont fait auriont nous $\mathrm{pu}$ nous dispenser de les distribuer aux sauvages nos alliez et a ceux qui nous les auriont pris, ce n'est done que ceux qui ont esté aretez aux environs du fort Cataracouy, lesquels sont bien Iroquois naturels, mais pour la plus part des villages du nort du lac ontario ou il y en avoit de beaux et Grands que les Iroquois du sud dudit lac ont obligé de se joindre a eux, ce qui a commencé à les grossir et a depeupler cette coste du nort, nostre Interest seroit de repeupler ces villages par ce qu'ils seroient plus sous nostre main.

Dans le nombre des prisonniers il y en a quelques uns que je ne dois point vous envoyer estans proches parens de nos sauvages Chrestiens, outre qu'il y en a du village des Onontaguez que nous devons menager pour tacher de les desunir des sonontouans et pour nous en servir pour negocier si nous en avons besoin.

Comme je ne sçay encore aucunes nouvelles de mouvemens des Iroquois je souhaiterois fort de ne me pas defaire de tous ces prisonniers cepant [sic] Monseigneur comme vous les desirez je me contanteray de retenir ceux que je croiray me pouvoir estre utile et que ne seront point coupables de tous les desordres des autres, si cependant Mgr vous voulez bien les retenir en lieu d'ou on les peut retirer en cas de besoin et que dans la suite on puisse venir à un accomodement general je croy que ce sceroit une chose tres utille au pays, a l'esgard de toutes leurs femmes et enfans Je les ay distribuez dans toutes 
nos missions de la Colonie, tous les hommes, femmes et enfans se sont fait batiser temoignans joye de cette occasion. Il reste a scavoir si c'est de bonne foy. ${ }^{46}$

Selon le Père Beschefer, s.j., trente-six Iroquois furent envoyés en France, ${ }^{47}$ et Denonville écrivit: «J'ay cru devoir aveuglement obeir aux ordres de Sa Majesté en renvoyant tous les prisonniers comme j'ay fait. ${ }^{48}$ Lorsque les prisonniers arrivèrent en France le ministre assura Denonville qu'ils seraient bien traités et il lui ordonna de capturer, dans le même but, encore autant d'Iroquois qu'il le pourrait dans les prochaines campagnes. ${ }^{49}$ A la même époque, le ministre donna instructions à l'intendant des galères de Marseille de ne pas les tenir enchâ̂nés et de leur donner des rations supplémentaires. Des dispositions furent aussi prises pour que l'instruction religieuse leur fût donnée. ${ }^{50}$ Nous devons aussi ajouter que les conditions sur les galères n'étaient pas aussi mauvaises qu'on l'imagine généralement. Les rameurs devaient être maintenus en bonne santé et pleins de vigueur sans quoi ils auraient été inutiles; et comparativement à la façon dont les Iroquois traitaient leurs propres prisonniers, ces galériens n'étaient pas maltraités. Denonville, cependant, s'inquiétait beaucoup du traitement qu'ils recevaient. En août 1688 il rappela au ministre qu'il faudrait renvoyer les prisonniers au Canada avant qu'un accord de paix pût être conclu avec les Iroquois. Quoiqu'il sût, disait-il, qu'au début ils avaient été bien traités, on ne lui avait pas moins rapporté qu'à cet égard les ordres du ministre n'avaient pas été, par la suite, observés très fidèlement et que les prisonniers avaient été contraints de marcher nu-pieds. Denonville craignait beaucoup qu'ils ne meu-

46 Denonville au Ministre, Mtl, 25 aoust 1687, op. cit.

47 R. P. Beschefer à M. Cabart de Villermont, Qué., 19 sept. 1687,

48 Paris, Archives Nationales, Colonies, Séries C11A, IX: 134-135, Memoire de l'Estat Present des affaires de Canada, Denonville, 27 oct. 1687. $49 \mathrm{Ibid}$., Séries B, XV: 26, Ministre à M. de Denonville, Versailles 8 mars 1688.

50 Collection de Documents Relatifs à l'Histoire de la Nouvelle-France, (Québec, 1883), I: 426, Ministre à M. l'Intendant des Galères, à Marseille, 1688; 394, Lettre du Ministre au R. P. Leroux à Marseille, Paris, 26 mars 1687. 
rent tous de mauvais traitement, ce qui rendrait très difficiles, insistait-il, les futurs pourparlers avec les Cinq Nations. ${ }^{51}$ Il fit suivre cette lettre d'une requête particulière: il voulait le renvoi des galériens iroquois en Nouvelle-France et souhaitait que Joseph Le Moyne de Sérigny, un officier de la Marine française qui parlait la langue iroquoise, les accompagnât à Rochefort. Il demanda aussi qu'ils fussent convenablement vêtus et traités avec douceur - «... les caresser un peu, quelques rubans et galons leur fera plaisir...». Ainsi s'exprime le commis qui résuma la dépêche de Denonville pour le ministre. ${ }^{52}$ En octobre de l'année suivante, 1689, les treize prisonniers survivants revinrent à Québec avec le convoi qui emmena Frontenac, Callières et l'expédition manquée destinée à la conquête de la Nouvelle-York. ${ }^{53}$

Selon Garneau les galériens iroquois furent renvoyés au Canada par Louis XIV. Le Roi, ajoute l'historien, «... desavoua la conduite du gouverneur... le droit des gens et le caractère sacré d'ambassadeur avaient été violés. Louis XIV s'empressa de renvoyer les captifs pour détruire l'effet d'une si fâcheuse méprise ... $\gg^{54}$ Affirmation erronée. Ni Louis XIV, ni le ministre ne désapprouvèrent la conduite de Denonville; ${ }^{55}$ de fait, le

51 Paris, Archives Nationales, Colonies, Séries C11A, X: 70, Denonville à Seignelay, 10 aoust 1688 .

52 Ibid., 105, Denonville au Ministre, 6 nov. 1688; 74, M. de Denonville, Mtl, 10 aoust 1688; ibid., Séries F3, Moreau de St-Méry, II : 237-242, Memoire succinct... a Monseigneur de la part de Denonville du $10^{\mathrm{e}}$ Aoust 1688.

53 Ibid., XI: 7, Relation de ce qui s'est passé de plus remarquable en Canada depuis... Nov. 1689 jusqu'au Mois de Nov. 1690. Monseignat; voir aussi, Collection de Documents Relatifs à l'Histoire de la NouvelleFrance, I: 454. Les noms de vingt-et-un galériens iroquois qui furent envoyés à Rochefort pour retourner au Canada, y sont inscrits. Les Iroquois déclarèrent eux-mêmes que vingt-huit de leurs guerriers avaient été capturés par les Français et plus tard ils racontèrent que treize prisonniers avaient été renvoyés de France au Canada, «les autres, au nombre de 23, étaient tous morts de maladie 》. Voir Documents Relating to the Colonial History of New York, III: 557-561; Albany, State Archives of New York, New York Colonial Manuscripts, XXXVI: 19, Albany Convention, Dec. 27, 1689.

54 François-Xavier Garneau, op. cit., I: 329.

55 Il est même fort douteux que Louis XIV sût qu'il y avait des Iroquois sur ses galères; il avait à s'occuper d'affaires bien plus importantes. Les dépêches données comme venant de lui et qui portaient sa signature, ne furent pas écrites par lui; elles furent écrites par un secrétaire d'après quelques notes concises fournies par le ministre et la signature de Louis 
ministre lui ordonna de capturer et d'envoyer en France d'autres prisonniers de ce genre. ${ }^{56}$ Que les droits sacrés des ambassadeurs aient été violés, les documents ne permettent pas d'affirmer telle chose; ils tendraient plutôt à contredire cette hypothèse. Enfin Louis XIV ne se hâta pas de renvoyer les prisonniers au Canada; ils ne furent renvoyés que presque deux ans après leur arrivée en France et, d'ailleurs, seulement à la demande pressante de Denonville et de personne autre.

Voici une autre affirmation plus récente: «Pendant la nuit du 5 août 1689 , le village de Lachine fut complètement détruit et ses habitants tués ou faits prisonniers. Il en fut ainsi de La Chesnaye et de d'autres petits établissements. Les Iroquois tentaient évidemment de faire autant de prisonniers qu'ils le pouvaient, afin de recouvrer leurs propres parents qui étaient encore détenus en France. Ils réussirent dans leur dessein. Le Roi ordonna finalement que les captifs soient renvoyés chez eux. ${ }^{57}$ Hypothèse fort loin de la vérité historique. Les captifs iroquois pouvaient difficilement avoir été renvoyés par suite d'un événement qui n'avait pas encore eu lieu; les vaisseaux qui les ramenèrent partirent de La Rochelle le 23 juillet, l'agression contre Lachine survint deux semaines plus tard. ${ }^{58}$ De plus, le village de Lachine ne fut pas complètement détruit; des soixante-dix-sept maisons qui s'y trouvaient, cinquante-six furent incendiées; et les habitants ne furent pas tous tués ou capturés; la majorité gagna les forts environnants ou Montréal. ${ }^{59}$ Le

fut apposée non par Louis mais par Monsieur Rose, son premier secrétaire qui avait la plume, à qui il appartenait de contrefaire la signature de Louis sur les documents. Parfois on est porté à soupçonner que plusieurs de ces dépêches, expédiées sous la signature de Louis - et aussi sous celle du ministre -, furent écrites par des commis, puis envoyées sans que le ministre se souciât de les lire, ou du moins de les lire très attentivement; cela semble avoir été particulièrement vrai de Seignelay et de Pontchartrain. En un mot, la méthode était alors à peu près la même qu'elle l'est aujourd'hui dans les milieux ministériels. On se gardera donc bien d'affirmer que Louis XIV écrivit ceci, ou que le Roi ordonna cela; il serait plus exact d'affirmer que le ministre, ou que le gouvernement de Versailles, le fit. 56 Voir précédemment la note 49 , au bas de la page.

57 Preston \& Lamontagne, op. cit., 51.

58 Paris, Archives Nationales, Colonies, Séries C11D, II: 134, Le $\mathbf{S}^{\mathrm{r}}$ Saccardy au Ministre, La Rochelle, 3 jan. 1690.

59 Désiré Girouard, L'Ancien et le Nouveau Lac Saint-Louis, (Montréal, 1895), 124, 135. 
massacre de La Chesnaye n'eut pas lieu à cette époque mais le 13 novembre, un mois après que Frontenac eut remplacé Denonville comme gouverneur. ${ }^{60}$ On ne peut pas dire non plus que le massacre de Lachine et les longues années d'hostilités qui suivirent eurent pour cause la capture et l'envoi en France de ces Iroquois par Denonville. En 1688, les Iroquois avaient accepté un accord général de paix que l'on devait ratifier l'été suivant. Une des conditions de cette entente voulait que Denonville rendît tous les prisonniers, y compris ceux qui étaient en France. ${ }^{61}$ Qu'ils n'eussent pas été renvoyés plus tôt à Québec, Denonville n'y était pour rien; et, même l'eussent-ils été, que l'on n'eût pas évité les hostilités avec les Iroquois. Sur ces entrefaites, l'Angleterre avait déclaré la guerre à la France. Antérieurement à ceci, les deux gouvernements avaient conclu un traité de neutralité en Amérique du Nord; en même temps toute la question de leurs différends dans cette région était débattue et tranchée. ${ }^{62} \mathrm{Le}$ gouverneur Dongan et Andros, son successeur, cherchèrent alors à empêcher les Iroquois d'attaquer les établissements français; ${ }^{63}$ mais lorsque la révolution éclata en Angleterre et que les colonies anglaises apprirent, avant la Nouvelle-France, l'état de guerre entre les deux pays, ${ }^{64}$ rien ne pouvait empêcher les Iroquois de lancer tous leurs effectifs contre les Français; ils croyaient, en effet, pouvoir compter sur l'efficace appui militaire des Anglais. Ainsi le 16 août, deux newyorkais, Messieurs Philips et Van Cortland, déclaraient dans une lettre à M. Blathwayt, le secrétaire du Conseil privé: «Les nations indiennes des cantons en

60 Paris, Archives Nationales, Colonies, Séries C11A, X: 207, Frontenac au Ministre, Qué., 17 nov. 1689.

61 Ibid., Séries F3, Moreau de St-Méry, II: 237-242, Memoire succinct... a Monseigneur de la part de Denonville du $10^{\mathrm{e}}$ Aoust 1688.

62 Ibid., Séries B, XV: 7-8, Lettre du Roy à M. le marquis de Denonville, Versailles, 8 jan. 1685; Calendar of State Papers Colonial, America \& West Indies 1685-1688, 368, Memorandum.

63 Documents Relating to the Colonial History of New York, III: 396, Gov. Dongan's rpt. on the state of the Province... [1687]; 558, Proceedings between Gov. Andros \& the Five Nations ... Albany, Sept. 19, 1688.

64 Ibid., 591, Stephen Van Cortlandt to Gov. Andros, N. Yorke, July 9, 1689 ; Calendar of State Papers Colonial, America \& West Indies, 1689-1692, 22, Earl of Shrewsbury to Lord Howard of Effingham, Whitehall, April 19, 1689; Paris, Archives Nationales, Séries C11A, X: 226-227, Champigny au Ministre, Mtl, 6 juillet 1689. 
amont d'Albany ayant entendu parler de guerre entre l'Angleterre et la France sont allés attaquer les habitants du Canada. » ${ }^{65}$

La plus grave erreur de Denonville fut d'obéir à l'ordre d'envoyer en France tout Iroquois fait prisonnier. Même s'il garda quinze de ces prisonniers, il savait fort bien qu'il aurait besoin, pour les futurs pourparlers avec les Iroquois, de quelquesuns, sinon de tous ceux qu'il avait envoyés outre-mer. Il ne pouvait ignorer non plus qu'il y avait très grand risque que plusieurs, et peut-être tous les prisonniers, succombent à la maladie et aux privations, soit en route vers la France, soit sur les galères, soit au retour. Il choisit néanmoins, pour employer sa propre expression, d'obéir aveuglément à l'ordre reçu. Indépendamment de considérations humanitaires, c'était une grave erreur de jugement et une erreur que Denonville reconnut implicitement lorsqu'il demanda au ministre de renvoyer les prisonniers au Canada. L'erreur compte peu cependant dans les événements du temps. Ces prisonniers même renvoyés plus tôt, ou point du tout envoyés en France, les Iroquois auraient sûrement et quand même déclanché leurs attaques contre les établissements français, dès lors qu'ils apprirent la déclaration des hostilités entre l'Angleterre et la France.

Les faits le démontrent abondamment: Denonville a été durement et injustement blâmé. On ne peut affirmer catégoriquement qu'il captura les Iroquois dans le but prémédité de les envoyer aux galères; la preuve indique plutôt qu'il les captura au cours d'opérations militaires, afin de protéger son armée et ses opérations. Les moyens employés par Champigny - non par Denonville - pour capturer quelques-uns de ces prisonniers étaient de qualité douteuse, pour ne pas dire plus; la preuve, cependant, semblerait indiquer qu'à cette époque on n'avait pas l'intention de leur faire aucun mal sérieux, mais simplement de s'assurer qu'ils ne causeraient aucun tort à l'armée française. On ne peut non plus affirmer que Denonville s'empara traîtreusement, au Fort Frontenac, d'un groupe d'ambassadeurs iroquois ; plusieurs documents réfutent cette assertion. L'historien peut

65 Documents Relating to the Colonial History of New York, III : 608, Messrs Philips \& Van Cortland to Secretary Blathwayt, Aug. 5, 1689. 
tenir compte, sans aucun doute, de preuves qu'un tribunal rejetterait. Encore incombe-t-il à ceux qui condamneraient Denonville en ces agissements de fournir la preuve de leur condamnation. Il ne devrait pas être condamné, comme il l'a été, pour des motifs que, selon toute évidence, il paraît bien n'avoir jamais eus; pour des choses qu'il n'a pas faites; ni pour des conséquences qui provenaient d'événements complètement indépendants de sa volonté et avec lesquels il n'avait rien eu à voir.

W. J. ECCLES,

Associate Professor of History,

Department of History,

University of Alberta,

Edmonton, Alberta.

Articles à paraître :

JEANNE GRÉGOIRE, Les Acadiens dans une seconde patrie: la Louisiane.

JEANNE GRÉGOIRE, Louisiane et langue française.

GASTON CARRIÈRE, o.m.i., Projets de préfectures apostoliques pour le nord-est du Canada.

L. JoRE, Un Canadien, gouverneur du Sénégal, Louis Le Gardeur de Repentigny, 1721-1786.

Roland LAMONTAGNE, La construction $d u$ fort Saint-Jean, $1748-1749$.

Robert Le Blant, Notes sur Jean de Garibal, associé de Notre-Dame-de-Montréal, vers le 24 juillet 1619 - 17 juillet 1667 .

Antonio DRolet, La bibliothèque du Collège de Québec, 1632-1800.

YVoN THÉRIAULT, Essai d'économie humaine: Industrialisation des Trois-Rivières. 